Abstract EPV115/\#333 Table 1 Pre-operative characteristics and ivestigations of patients with UCS

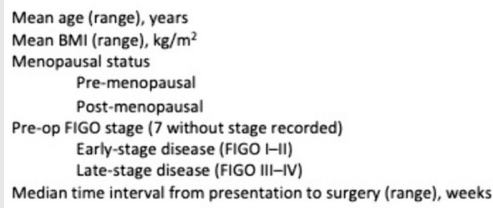

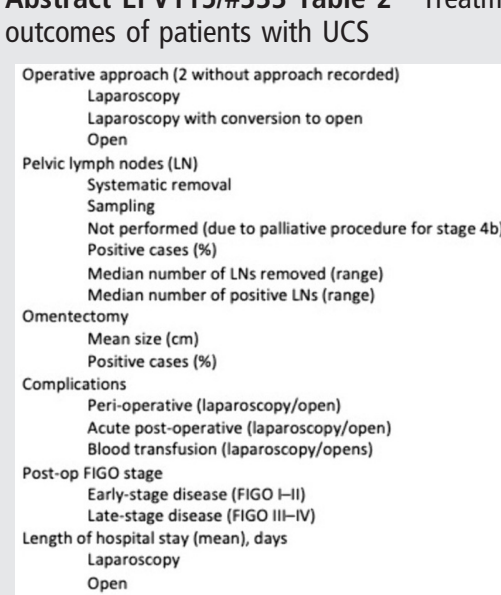
outcomes of patients with UCS

Abstract EPV115/\#333 Table 2 Treatment modalities and

Conclusions UCS presented with almost double the incidence rate previously described. The overall accuracy of pre-operative staging was $81 \%$. Our analysis showed that $86 \%$ of cases were managed laparoscopically, with more favourable peri-operative and post-operative profiles. Despite the lack of management guidelines, we stress the importance of urgent surgical treatment for UCS, in the form of total hysterectomy, bilateral salpingo-oophorectomy, systematic bilateral pelvic lymph node dissection and omentectomy. Data on adjuvant treatment, recurrence and survival is currently under analysis.

\section{EPV116/\#34 HIGH GRADE ENDOMETRIAL CARCINOMA LIMITED TO THE ENDOMETRIUM OR A POLYP. IS ADJUVANT TREATMENT NECESSARY?}

L Dallaire Nantel*, MC Renaud, A Sebastianelli, J Grégoire, M Plante. Hotel Dieu de Quebec, Gynecology Oncology, Quebec, Canada

10.1136/ijgc-2021-IGCS. 186

Objectives High grade endometrial carcinoma limited to the endometrium or a polyp is a rare clinical entity. Currently there is no consensus on standard treatment. Thus, the goal of this study was to evaluate the clinical outcomes of patients with type II endometrial carcinoma without myometrial infiltration or limited to a polyp.

Methods We retrospectively identified type II endometrial carcinoma with spread limited to the endometrium or a polyp from April 2013 to November 2017. Medical records were reviewed for the following information: age at diagnosis, characteristics of patients, type of surgery, histology, stage according to FIGO 2009 classification, adjuvant treatments, and site of recurrence. Descriptive statistics and the KaplanMeier estimate were used for analysis.

Results Twenty-six patients with a type II stage IA adenocarcinoma were included. All were surgically staged with total hysterectomy, salpingo-oophorectomy and lymph nodes assessment. The median age at diagnosis was 69 years. All patients had either disease limited to the endometrium (61.5\%) or a polyp (38.5\%). Only four patients had lymphovascular space invasion (16.5\%). Median follow up was 44 months (2-75 months). Most patients did not receive adjuvant treatment after surgery (73\%). Three patients (11.5\%) experienced recurrences 15, 21 and 55 months after surgery. Following systemic treatment all are alive and free of disease. The 3-year progression free survival and overall survival were $91 \%$ and $100 \%$ respectively.

Conclusions Based on our data, expectant management with surveillance alone following surgery appears to be safe for patients with high-grade endometrial carcinoma limited to a polyp or the endometrium without myometrial invasion.

\section{EPV117/\#36 PD-L1 IS A TUMOR SUPPRESSOR IN AGGRESSIVE ENDOMETRIAL CANCER CELLS AND ITS EXPRESSION IS REGULATED BY MIR-216A AND LNCRNA MEG3}

P Dong*. Hokkaido University, Department of Obstetrics and Gynecology, Sapporo, Japan

\subsection{6/ijgc-2021-IGCS.187}

Objectives Poorly-differentiated endometrioid adenocarcinoma and serous adenocarcinoma represent an aggressive subtype of endometrial cancer (EC). Programmed death-ligand-1 (PD-L1) was known to exhibit a tumor cell-intrinsic function in mediating immune-independent tumor progression. However, the functional relevance of tumor cell-intrinsic PD-L1 expression in aggressive EC cells and the mechanisms regulating its expression remain unknown.

Methods PD-L1 expression in 65 EC tissues and 18 normal endometrium samples was analyzed using immunohistochemical staining.

Results Positive PD-L1 expression was identified in $84 \%$ of benign cases but only in $12 \%$ of the EC samples, and the staining levels of PD-L1 in EC tissues were significantly lower than those in the normal tissues. Higher PD-L1 expression predicts favorable survival in EC. Ectopic expression of PD-L1 in aggressive EC cells results in decreased cell proliferation and the loss of mesenchymal phenotypes. Mechanistically, PDL1 exerts the anti-tumor effects by downregulating MCL-1 expression. We found that PD-L1 levels in aggressive EC cells are regulated by miR-216a, which directly targets PD-L1. We further identified a mechanism whereby the long non-coding RNA MEG3 represses the expression of miR-216a, thereby leading to increased PD-L1 expression and significant inhibition of cell migration and invasion.

Conclusions These results revealed an unappreciated tumor cell-intrinsic role for PD-L1 as a tumor suppressor in aggressive EC cells, and identify MEG3 and miR-216a as upstream regulators of PD-L1. 